\author{
В. А. Шевченко, к.т.н., доиент, \\ зав. кафедрой технической эксплуатаиии судов \\ e-mail: vash4891@gmail.com \\ Национальный университет «Одесская морская академия» \\ ул. Дидрихсона, 8, г. Одесса, 65000, Украина
}

\title{
ОПТИМИЗАЦИЯ УПРАВЛЕНИЯ ЭЛЕКТРОСТАНЦИЕЙ ИЗ УСЛОВИЯ МИНИМУМА РАСХОДА ТОПЛИВА И С УЧЕТОМ МЕТЕОУСЛОВИЙ
}

В работе рассмотрено решение задачи повышения эффективности управления электростанцией по критерию минимального расхода топлива с учетом метеоусловий. Проанализированы режимы работы электростанций современных судов и их средняя загрузка. Предложены выражения для определения суммарного коэффициента избытка воздуха двигателей в судовой электростанции с учетом расхода влажного и сухого воздуха, определяемого по номограммам, приведенным в работе. Получены выражения, позволяющие определить оптимальный коэффициент избытка воздуха двигателя внутреннего сгорания, соответствующий максимальному КПД. Разработаны алгоритмы управления электростанцией с учетом минимума расхода топлива путем варьирования коэффициентом избытка воздуха и топливоподачей. Выполненные исследования позволяют установить правила функционирования системы оптимального управления электростанцией при изменении нагрузки, технического состояния ГА и условий окружаюшей среды.

Ключевые слова: управление электростаниией, коэффициент избытка воздуха, синтез алгоритмов, расход топлива, оптимизачия.

Введение. Эксплуатируемые на современных судах автоматизированные электроэнергетические системы характеризуются низким коэффициентом загрузки установленной мощности и, как следствие, невысокой экономичностью, что объясняется не только комплектацией электростанции, но и несовершенной организацией режимов работы. До сих пор решение рассматриваемых задач производят, как правило, с помощью приближенных, а зачастую достаточно грубых способов [1], стремясь в большей мере к увеличению только одного критерия - резервирование ЭЭС в основных режимах работы судна.

Анализ последних источников исследований и публикаций. При расчете мощности и допустимых значений загрузки электростанции по режимам исходят обычно из усредненных либо предельных значений неуправляемых переменных (запроса загрузки, температуры, давления, влажности среды и т. д.), не учитывая возможный диапазон их изменения, что приводит к снижению технико-экономических показателей, о чем свидетельствует целый ряд публикаций [2-5].

Рассмотренные в [6] задачи управления электростанцией по принципу гибких порогов загрузки вызывают необходимость более детальной проработки вопросов оптимизации, связанных с ресурсосбережением и минимизацией расхода топлива. Особое внимание при этом обращается на необходимость учета метеоусловий в расчете верхних уровней загрузки дизелей во избежание их термических перегрузок. Отмечается $[1,7]$ сложный характер воздействия метеоусловий на рабочие параметры дизелей, отличающихся многообразием конструкций. Предельная нагрузка дизелей начинает снижаться при повышении температуры окружающего воздуха более $32^{\circ} \mathrm{C}$, влажности более $60 \%$ и снижениях барометрического давления ниже 101,3 кПа [4]. Воздействие этих факторов в современных системах учитывается выборочно, в то время как они действуют взаимосвязано.

Например, завод-изготовитель "WartsilaSulzer" [8] указывает в паспорте дизеля, что нормальными параметрами среды являются: атмосферное давление 960 мбар (720 мм рт. ст.), температура воздуха $20^{\circ} \mathrm{C}$, влажность $70 \%$, и предупреждает о том, что всякое отклонение этих величин, особенно температуры всасывающего воздуха и давления, может существенно изменить характеристики дизеля. 
Наиболее характерными изменениями при плавании судна в тропических условиях являются: уменьшение мощности, увеличение удельного расхода топлива и температуры выпускных газов, вызванные уменьшением весового заряда воздуха и повышением его начальной температуры [3].

Вызвано это тем, что при уменьшении весового заряда воздуха $\rho_{\mathrm{k}}$ уменьшается коэффициент избытка воздуха $\alpha=k \eta_{\nu} \rho_{\mathrm{k}} / g_{\text {ц }}$, где $\eta_{v}$ - коэффициент наполнения цилиндра, a $g_{ц}$ - цикловая подача топлива. В свою очередь, снижение $\alpha$ вызовет уменьшение индикаторного $\eta_{i}$ и, как следствие, эффективного $\eta_{e}$ КПД двигателя $\eta_{e}=f(\alpha)$. В результате вращающий момент дизеля $M=k g_{\text {ц }} \eta_{e}$ начнет снижаться, а регулятор частоты с целью стабилизации частоты тока начнет увеличивать подачу топлива $g_{\text {ц }}$, чем вызовет перегрузку по среднему индикаторному давлению. При этом температура выпускных газов начнет возрастать. Чрезмерное повышение температуры выпускных газов вынуждает судовых специалистов уменьшать цикловую подачу топлива, что приводит к суммарному уменьшению мощности ГА.

Проведенные исследования режимов работы шестиагрегатной электростанции судов типа "HAI YANG SHI YOU 702" показали, что при плавании судна в различных широтах, в том числе и тропической зоне, наблюдаются колебания метеоэлементов в широком диапазоне $\left(t^{\circ}=20 \div 45^{\circ} \mathrm{C} ; \varphi=60 \div\right.$ $85 \% ; P_{a}=750 \div 780$ мм рт.ст.), что приводит к изменению предельно допустимой, по условиям термической нагрузки, мощности от 735 до 604 кВт (на 18\%). Частые колебания нагрузки и параметров среды в течение суток делают для обслуживающего персонала трудноосуществимым решение задачи по оптимизации управления составом ГА. Поэтому, во избежание риска, в отношении термической перегрузки приходится держать включенным достаточно большой запас генерируемой мощности. Например, в ходовом режиме вместо возможных четырех задействуют, как правило, пять ГА, длительно работающих при малых и средних нагрузках, что приводит к снижению механического КПД и, как следствие, уменьшению эффективного $\eta_{e}=\eta_{i} \eta_{m}$ КПД.
Анализ условий эксплуатации двигателей электрогенераторов по транспортному флоту [9] показывает, что средняя загрузка судовых электростанций составляет $30 \div 35 \%$ общей номинальной мощности генераторов. На судах отдельных типов дизель-генераторы в течение почти всего рабочего времени (90\%) загружены менее, чем на половину. Длительная работа двигателей с пониженной нагрузкой приводит к отложениям на продувочных и выхлопных окнах, клапанах, газотурбонагнетателях, выхлопных коллекторах и трубопроводах, что снижает экономичность двигателя. Известны случаи выхода из строя мотылевых и рамовых подшипников двигателей из-за продолжительной работы на малых нагрузках. На судах типа DSV "Bar Protector" при работе одного ДГ с нагрузкой 270 кВТ вместо двух ДГ, работающих параллельно с нагрузкой менее $50 \% N_{e_{\text {ном }}}$, суточная экономия дизельного топлива достигнет $0,8 \mathrm{~T}$, а циркуляционного масла - 8-10 л при сохранении ресурса ДГ.

Таким образом, устранение этих недостатков позволило бы в целом по флоту получить существенный технико-экономический эффект.

Цель работы - решение задачи управления электростанцией из условия минимума расхода топлива, разработка алгоритмов расчета и непрерывного контроля допустимых нагрузок дизелей, а также алгоритмов автоматического регулирования параметров работы двигателя в соответствии с изменениями параметров среды.

Изложение основного материала. Международным конгрессом двигателестроения и специальным комитетом СЭВ рекомендована методика, в основу которой положены формулы К. Циннера [10] при определении зависимости изменения индикаторной мощности, где индикаторная мощность:

$$
k=\frac{N_{i \mathrm{~T}}}{N_{i_{\mathrm{H}}}}=\left(\frac{D-P_{\Pi}}{99}\right)^{m}\left(\frac{293}{T}\right)^{n}\left(\frac{293}{T_{w}}\right)^{q},
$$

где $N_{i 3}, N_{i o}-$ индикаторные мощности в текущих и нормальных условиях соответственно; $D$ - барометрическое давление, кПа; $P_{\Pi}-$ парциальное давление водяных паров, кПа; $T$ температура воздуха на всасывании, ${ }^{\circ} \mathrm{K} ; T_{w}-$ температура охлаждающей воды, ${ }^{\circ} \mathrm{K}$.

Парциальное давление определяется по i-d диаграмме влажного воздуха (рис. 1), в 
функции температуры и влажности среды; показатели степени $m, n, q$ принимаются в зависимости от нормальных значений суммарного коэффициента $\alpha_{\text {с }}$ избытка воздуха

$$
\begin{aligned}
& m=\left\{\begin{array}{l}
0,1, \text { если } 1,7<\alpha<2,1 \\
0,3, \text { если } \alpha<1,7
\end{array},\right. \\
& n=\left\{\begin{array}{l}
0,2 \eta_{\mathrm{M}}, \text { если } \alpha>2,1 \\
0,35 \eta_{\mathrm{M}}, \text { если } 1,7<\alpha<2,1, \\
0,55 \eta_{\mathrm{M}}, \text { если } \alpha<1,7
\end{array}\right. \\
& q=\left\{\begin{array}{l}
0,2 \eta_{\mathrm{M}}, \text { если } \alpha>2,1 \\
0,3 \eta_{\mathrm{M}}, \text { если } 1,7<\alpha<2,1 . \\
0,6 \eta_{\mathrm{M}}, \text { если } \alpha<1,7
\end{array}\right.
\end{aligned}
$$

С учетом механического КПД $\eta_{\mathrm{M}}$ коэффициент пересчета $k_{R C L}$ мощности будет определяться формулой

$$
k_{R C L}=\left(k-0,7(1-k) / \eta_{\mathrm{M}}-1,\right.
$$

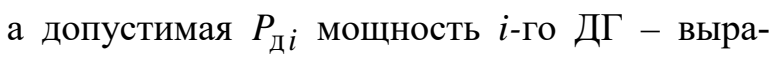
жением $P_{\text {дi }}=k_{R C L} \cdot P_{\mathrm{H}}$, где $P_{\mathrm{H}}-$ номинальная мощность двигателя.

Расчеты показывают, что при изменении температуры воздуха от 27 до $62^{\circ} \mathrm{C}$ значения $k_{R C L}$ изменялись от 0,96 до 0,88 , а при изменениях трех параметров $(T, D, j)$ - от 0,93 до 0,85. При этом температура охлаждающей воды изменялась в пределах $25-35^{\circ} \mathrm{C}$.

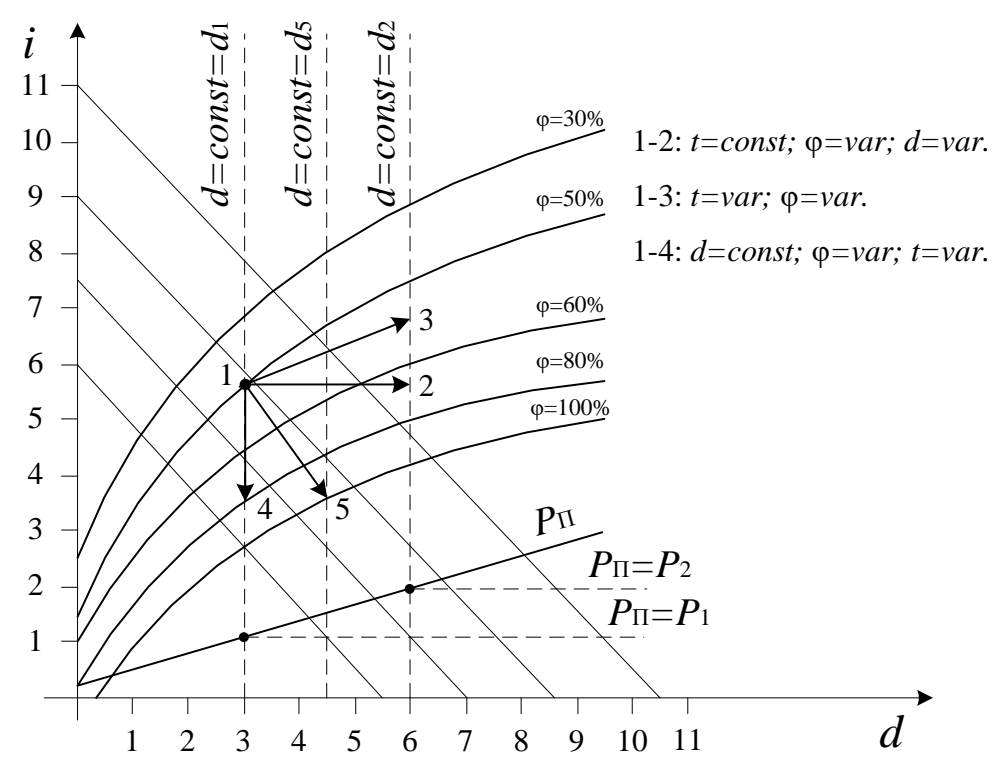

Рис. 1. Влияние изменения относительной влажности на парциальное давление

Таким образом, нами установлен закон определения $k_{R C L}$, использование которого в алгоритмах работы [6] придает гибкость в порождении предикатов $P H(l), P D(l)$, что, в свою очередь, позволит эксплуатировать ГА в зонах, близких к заградительным характеристикам, и тем самым повысить коэффициент загрузки судовых электростанций.

Тем не менее, можно предложить еще один из способов дальнейшего совершенствования алгоритмов управления электростанцией путем поддержания оптимального режима работы двигателя, если в качестве критерия оценки режима принять [4] постоянство значения суммарного коэффициента избытка воздуха

$$
\alpha_{c}-\alpha_{o p t} \Rightarrow \min
$$

где $\alpha_{c}-$ текущее, $\alpha_{o p t}$ - оптимальное для установившегося режима работы дизеля значение коэффициента, при котором достигается максимально возможная мощность при минимальном расходе топлива и допустимой температуре выпускных газов. В судовых условиях суммарный коэффициент избытка воздуха можно определить [4] по показаниям приборов, пользуясь выражением

$$
\alpha_{c}=A_{1} \frac{P_{S}}{T_{S} G_{T}}\left(\frac{1}{1+1,61 d(i)}\right),
$$

где $A_{1}$ - константа, учитывающая конструктивные особенности дизеля, а также то, что 
произведение коэффициентов наполнения и продувки можно считать практически постоянным; $P_{S}$ и $T_{S}$ - давление и температура воздуха в турбонагнетателе; $G_{T}$ - расход топлива в единицу времени; $d(i)$ - влагосодержание воздуха в $i$-том установившемся режиме.

Эту взаимосвязь можно представить в виде номограммы (рис. 2), где расходы влажного и сухого воздуха связаны отношением

$$
\frac{G_{\mathrm{BC}}}{G_{\mathrm{BB}}}=\frac{1}{1+1,61 d_{i}} \text {. }
$$

Значение $d_{i}$ находят при помощи таблиц влажного воздуха или $i-d$ диаграммы (рис. 1).

Из номограммы следует, что, если при нагрузке ДГ $P(i)$ и расходе топлива $G_{T}^{A}$ остальные рабочие параметры будут характеризоваться набором $\left\{P_{S}(i), T_{S}(i), d_{i}\right\}$, то суммарный коэффициент избытка воздуха $\alpha_{C}(i)<\alpha_{\text {opt }}$ будет меньше оптимального значения, а расход сухого воздуха - на $\Delta G_{\mathrm{BC}}$ меньше нормы, что ухудшит процесс сгорания и приведет к росту температуры выпускных газов.

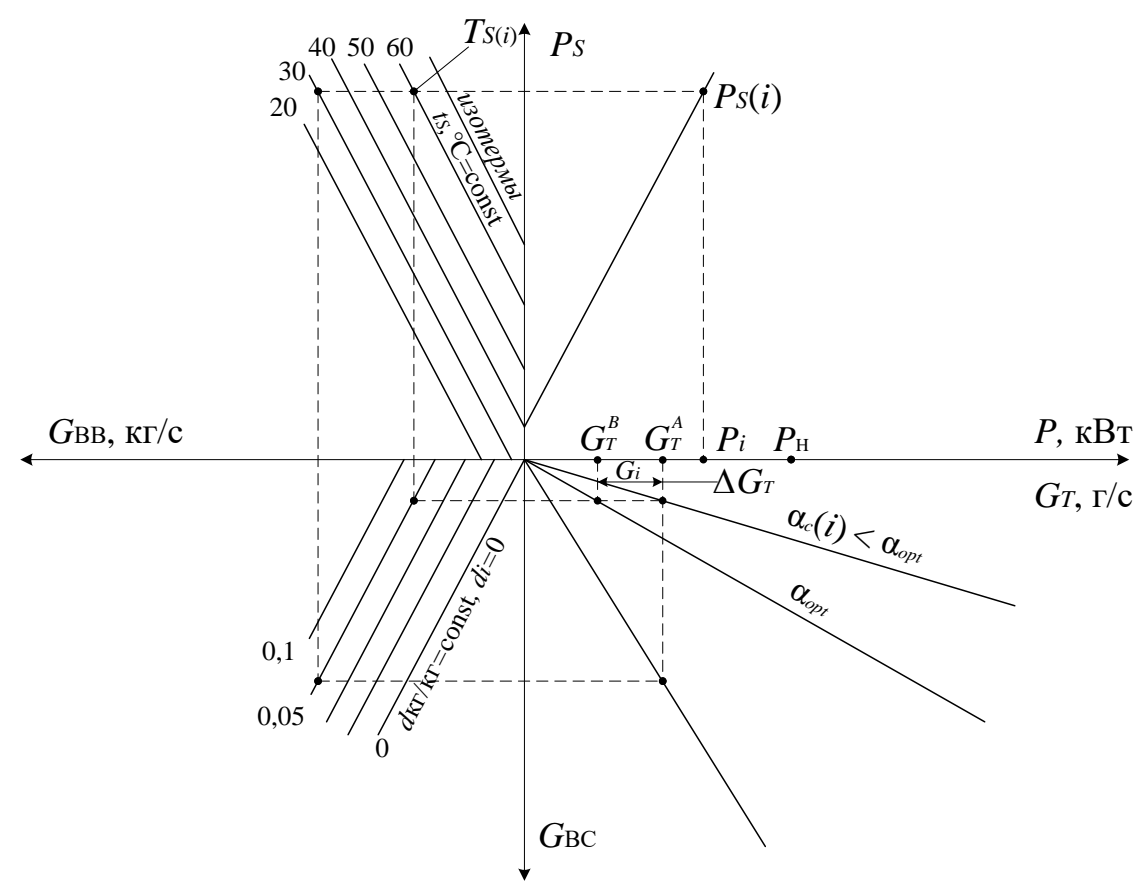

Рис. 2. Номограмма взаимосвязи расходов влажного и сухого воздуха

В этом случае регулировать режим работы дизеля можно двумя способами: уменьшением расхода топлива на величину $\Delta G_{T}$ путем перераспределения нагрузок между параллельно работающими ГА $P R S H$ или подключением накопителей ONACC или отключением второстепенных OFCNS для данного режима потребителя; снижением температуры наддувочного воздуха $T_{S}$ за счет увеличения потока охлаждающей воды в системе охлаждения наддувочного воздуха.

Следует отметить, что зона $\alpha_{c}>\alpha_{o p t}$ является также нежелательной, поскольку увеличение $\alpha_{c}$ относительно оптимального приводит к ухудшению процесса сгорания, увеличению потерь тепла с уходящими газами и уменьшению индикаторного КПД

Выполненные исследования позволяют синтезировать структуру алгоритма оптимизации PROPT режимов работы дизелей, если в качестве условия принять, что система содержит все необходимые датчики $\left\{P_{S}, P_{i}, T_{S}, G_{T}, \varphi, t^{\Gamma}\right\}$, где $j-$ влажность воздуха, $t^{\Gamma}$ - температура выхлопных газов, $\{S M W, S M F\}$ - исполнительные органы (сервомоторы), с помощью которых можно управлять подачей воды $S M W$ для охлаждения наддувочного воздуха и подачей топлива SMF. 
В качестве способа организации работы программы PROPT выберем способ прерывания с периодом дискретности $T_{\text {цо }}$ определяемым динамическими свойствами системы охлаждения наддувочного воздуха и дизеля как двигателя внутреннего сгорания.

Для облегчения процедуры логического программирования представим программу $P R O P T$ в виде совокупности $P R O P T=$ $\{S B H T P, S B A L F, S B S M, S B C N T\}$ подпрограмм, а именно подпрограммы $S B$ контроля высокой температуры $S B H T P$, расчета отклонений коэффициента избытка воздуха $S B A L F$, управления сервомоторами $S B S M$ и расчета длительности включения сервомотора $C N T$.

С учетом изложенного, запишем правила функционирования системы в виде следующих инструкций с использованием подхода, принятого нами в [11-13]:

while $T_{\mathrm{T}} d o$

PROPT,SBALF,SBHTP,SBSM,SBCNT od

while SBALF do: $\left(t_{i}^{\Gamma}-t_{\text {доп }}^{\Gamma}=\Delta t^{\Gamma}\right)$ if

$\Delta t^{\Gamma}>0$ then HTP

go to SBHTP else do:

$\left(\alpha_{c}(i)=f\left(P_{S}, P_{i}, T_{S}, G, \varphi, \Delta \alpha_{c}(i)-\alpha_{o p t}\right)\right.$

if $\left|\Delta \alpha_{C}(i)\right|>\Delta \alpha_{\text {доп }}$

then go to SBSM else fi od,

т. е. выполнение подпрограммы $S B A L F$ всегда начинается с контроля температуры выпускных газов $t^{\Gamma}$ и, если она выше нормы $(H T P=$ И), то осуществляется переход к SBHTP, иначе производится расчет $\alpha_{C}(i)$ и сравнение его с оптимальным значением $\alpha_{\text {opt }}$, если при этом отклонение выше допустимого, то переход к $S B S M$, иначе конец программы. правилу:

Подпрограмма BHTP выполняется по

while SBHTP do if HTP \& $\overline{W V O P N}$ then

ONSMW(INC) do $B_{W}=f\left(\Delta t^{\Gamma}\right)$ go to SBCNT

else if $H T P \& W V O P N \& D C F$

then ONSMF(DEC) do

$$
B_{F}=f\left(\Delta t^{\Gamma}\right) \text { go to } \operatorname{SCBNT}\left(B_{F}\right) \text { else fi od, }
$$

т. е., если температура выпускных газов выше нормы $(H T P=$ И) и клапан системы охлаждения наддувочного воздуха полностью не открыт $\overline{W V O P N}$, то: включается $O N$ сервомотор $S M$ в направлении увеличения INC подачи охлаждающей воды ONSMW(INC); рассчиты- вается время его работы $B_{W}$ в зависимости от превышения температуры и осуществляется переход к подпрограмме отсчета SBCNT времени работы сервомотора. Если же при повышенной температуре газов клапан воды окажется полностью открытым $W V O P N$ и на верхнем уровне (уровне координатора) принято решение DCF снизить температуру газов уменьшением подачи топлива, то включается $O N$ серводвигатель $S M F$ регулятора в направлении уменьшения подачи топлива ON$S M F(D E C)$, рассчитывается время его работы $B_{F}$ и осуществляется переход к подпрограмме отсчета временного интервала $\operatorname{SBCNT}\left(B_{F}\right)$, иначе конец подпрограммы.

Остальные $\{S B S M, S B C N T\}$ подпрограммы должны выполняться по следующим инструкциям:

while SBSM do if $\left(\Delta \alpha_{c}(i)<0\right) \& \overline{W V O P N}$ then ONSMW(INC) do $B_{W}=f(\Delta \alpha)$ go to $\operatorname{SBCNT}\left(B_{W}\right)$ else if $\left(\Delta \alpha_{C}(i)>0\right) \&$ $\overline{W V C L S} \& \overline{H T P})$

then $\operatorname{ONSMW}(D E C)$ do $B_{W}=f(\Delta \alpha)$ go to $\operatorname{SBCNT}\left(B_{W}\right)$ else if $\left(\Delta \alpha_{C}(i)<0\right) \&$

$W V O P N \& D C F$ then ONSMF $(D E C)$ do $B_{F}=$ $f(\Delta \alpha)$ go to $S B H T P$ else fi od

while $\operatorname{SBCNT}\left(B_{W}\right)$ do:

if $\left(\Delta B_{W}(t+1)-1(C N T)=0\right)$ then OFSMW else fi od;

while $\operatorname{SBCNT}\left(B_{F}\right)$

do: if $\left(\Delta B_{F}(t+1)-1(C N T)=0\right)$

then OFSMF else fi od,

т. е. при отклонениях суммарного коэффициента избытка воздуха в ту или другую сторону от оптимального включаются в соответствующем направлении сервомоторы, если есть возможность регулировки, в противном случае, когда клапан уже полностью открыт, регулировка осуществляется воздействием на подачу топлива, если есть на это разрешение координатора $(D C P=И)$. При превышении температуры выпускных газов процесс отправляется к подпрограмме SBHTP.

Выполнение подпрограмм SBCNT осуществляется путем сравнения результата вычитания из остатка числа, отображающего величину временного интервала $\Delta B(t+1)$, единицы $1(C N T)$ счета, которая, как правило, эквивалентна длительности основного такта $T_{\mathrm{TL}}$. 
Поддержание оптимального режима работы каждого ДГ путем регулирования $\alpha_{c}$ в какой-то мере способствует решению задачи минимизации расхода топлива, так как для текущего значения нагрузки система обеспечивает не только нормальный тепловой режим дизеля, но и максимальный КПД (рис. 3).

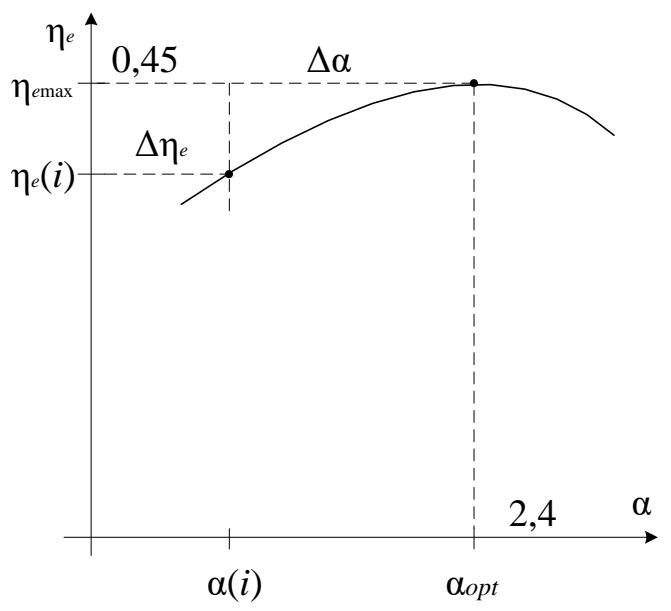

Рис. 3. Зависимость КПД от коэффициента избытка воздуха при 65\% нагрузке

Однако, в целом по электростанции целевая функция суммарного расхода топлива может не иметь глобального минимума:

$$
\begin{gathered}
F_{\Sigma}=\sum_{i=1}^{l} F(i) \rightarrow \min , F(i)=g_{e}(i) P_{\text {Д }}(i), \\
F_{\Sigma}=g_{e \Sigma} P_{\text {Д }} ; g_{e \Sigma}=\sum_{i=1}^{l} \lambda(i) g_{e}(i),
\end{gathered}
$$

где $F_{\Sigma}, F(i)$ - расходы топлива электростанции за час и $i$-го ГА; $g_{e \Sigma}, g_{e}(i)-$ удельные расходы топлива электростанции и $i$-го ГА; $P_{\Sigma}$ и $P_{\text {Д }}(i)-$ нагрузка электростанции и $i$-го $\Gamma$ А; $\lambda(i)=P_{Д}(i) / P_{\Sigma}-$ доля нагрузки $i$-го ГА.

Необходимым условием существования минимума $F_{\Sigma}$ является равенство нулю полного дифференциала $d g_{e \Sigma}$ :

$$
d g_{e \Sigma}=\sum_{i=1}^{l} \frac{\partial g_{e \Sigma}}{\partial P_{Д}(i)} d P_{Д}(i)=0 .
$$

Для получения экстремума функции $g_{e \Sigma}$ необходимо так распределить нагрузки, чтобы величины $\theta(i)$ каждого ГА в точках режима имели равные значения, т. е. $\theta(1)=\theta(2)=\ldots=\theta(i)=\ldots=\theta(l)$, где

$$
\theta(i)=\frac{\partial\left(P_{Д}(i) g_{e}(i)\right)}{\partial P_{Д}(i)}=g_{e}(i)+P_{Д}(i) \frac{\partial g_{e}(i)}{\partial P_{Д}(i)} .
$$

Достаточным условием оптимального распределения нагрузки является требование минимума $g_{e \Sigma} \rightarrow \min$. Это достаточное условие выполняется при $d^{2} g_{e \Sigma}>0$.

Если ГА имеют одинаковые нагрузочные характеристики, то для выполнения необходимого условия требуется в точках режимов работы электростанции распределить нагрузки равномерно. Для выполнения достаточности требуется выполнение набора условий:

$$
\begin{gathered}
\left\{2 \frac{\partial \theta}{\partial P_{Д}(i)}>0 ; 3\left(\frac{\partial \theta}{\partial P_{Д}(i)}\right)^{2}>\right. \\
\left.0 ; \ldots ; l\left(\frac{\partial \theta}{\partial P_{Д}(i)}\right)^{l-1}>0\right\},
\end{gathered}
$$

что возможно только в том случае, если все производные окажутся $\frac{\partial \theta}{\partial P_{Д}(i)}$ положительными.

Этот вывод является очень важным, поскольку дает возможность рассчитать по условиям оптимальности нижние пороги загрузки электростанции (рис. 4). В этом случае область работы генераторов (рис. 5), ограниченная допустимыми пределами, может возрасти, и ее выражение будет иметь вид

$$
P_{P D} \leq P_{\Gamma}(i) \leq\left(1-\frac{v_{1}}{l}\right) P_{\text {Д }} .
$$

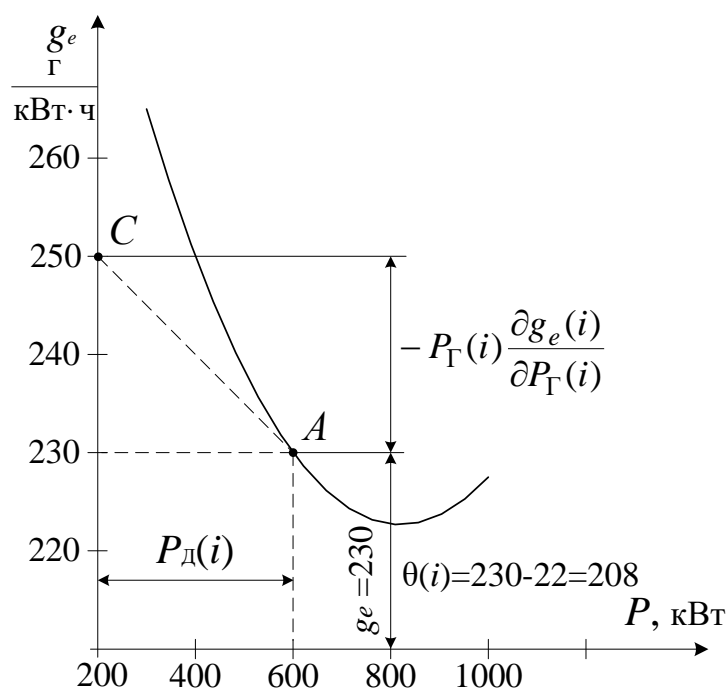

Рис. 4. К определению параметра $g_{e}$ 


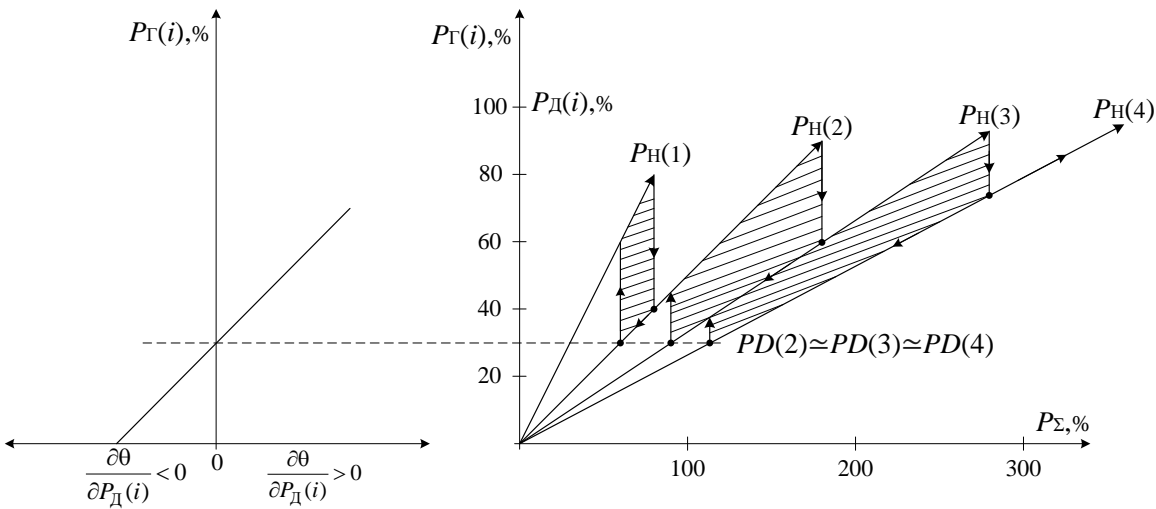

Рис. 5. Определение допустимого нижнего предела загрузки электростанции из условия оптимального распределения нагрузки

Однако в процессе эксплуатации нагрузочные характеристики первичных двигателей, как правило, изменяются, что может привести к существенному их разбросу и постановке задачи оптимизации режимов работы электростанции с учетом реальных нагрузочных характеристик.

Для решения задачи в такой постановке целесообразно использовать метод динамического программирования, обеспечивающий выбор наиболее вероятного (квазиоптимального), для определенного участка режима работы, состава ГА с соблюдением всех ограничений.

Для выполнения алгоритма динамического программирования должны быть построены эквивалентные характеристики $F_{\Sigma}^{Э}\left(P_{\Sigma}\right)$, которые представляют собой зависимости минимального расхода топлива агрегатов от их суммарной мощности, т. е.:

$$
F_{\Sigma}^{\ni}\left(P_{\Sigma}\right)=\min \left\{F_{i}(P(i))+F_{\Sigma-i}\left(P_{\Sigma}-P i\right)\right\} .
$$

Таким образом, для каждого дополнительного $P(i)$ нахождение $\min F_{\Sigma}^{Э}\left(P_{\Sigma}\right)$ сводится к нахождению глобального минимума функции $F_{i}(P(i))+F_{\Sigma-i}\left(P_{\Sigma}-P i\right)$, который может быть определен простым перебором. Одновременно с выбором состава ГА решается задача оптимального распределения нагрузок.

Выводы. Выполненные исследования позволяют установить следующие правила функционирования к алгоритму оптимального управления электростанцией при изменении нагрузки, технического состояния ГА и условий окружающей среды:

1. Формирование базы данных, определяющих количество работающих ГА, их техническое состояние, нагрузку, расход топлива и параметры окружающей среды.
2. Анализ нагрузочных характеристик агрегатов и формирование базы данных для расчетов по оптимизации режима.

3. Определение коэффициента пересчета мощности $k_{R C L}$ и корректировка верхних порогов загрузки из условий технического состояния и метеоэлементов.

4. Корректировка допустимых, по условиям оптимального распределения нагрузок, нижних порогов загрузки.

5. Определение суммарной нагрузки электростанции, анализ эквивалентных характеристик расхода топлива и выбор оптимального состава ГА по критерию минимума расхода топлива, а также с учетом ограничений по верхним и нижним порогам загрузки.

6. Выполнение операций пуска, синхронизации, перевода нагрузки и остановки ГА, связанных с формированием оптимального состава ГА.

7. Распределение нагрузок между параллельно работающими ГА по условиям оптимальности $F_{\Sigma} \min \left(P_{\Sigma}\right)$.

8. Выполнение программы PROPT оптимизации первичного двигателя электростанции по реализации критерия

$$
\left(\alpha_{c}-\alpha_{o p t}\right) \rightarrow \min .
$$

В процессе работы электростанции в установившемся режиме или в период смены состава по условиям оптимального управления могут возникать аварийные и предаварийные ситуации, вызванные выбегом контролируемых параметров за предельный уровень.

Для этих случаев должны быть предусмотрены алгоритмы формирования команд на ввод резервного и вывод аварийного ГА с учетом заданной очередности, разработке которых будут посвящены дальнейшие исследования. 


\section{Список литературы}

1. Mukund R. Patel. Shipboard electrical power systems. CRC Press, 2012. 370 p.

2. Kithsiri U. G., Senanayake1 N. S., Abeyweera Ruchira, Jayasuriya Jeevan. A model for estimation of de-rating in diesel engines used for power generation. International Journal of Energy Engineering. 2016. No. 6 (2). P. 3642. DOI: 10.5923/j.ijee.20160602.03.

3. Saber Hindren A., Al-Barwari Ramzi R. Ibraheem, Talabany Ziyad J. Effect of ambient air temperature on specific fuel consumption of naturally aspirated diesel engine. Journal of Science and Engineering. 2013. Vol. 1 (1). P. 1-7.

4. Mollenhauer Klaus, Tschoeke Helmut (eds). Diesel engine engineering hand book. Springer, 2010. ISBN-10: 3540890823.

5. McCrone A. K. L. Diesel plant operator's hand book. Pitman. ISBN-10: 0273409603.

6. Захарченко В. Н., Шевченко В. А. Решение задач управления судовой электроэнергетической установкой при изменении нагрузки. Судовые энергетические установки: науч.техн. сб. 2015. Вып. 36. С. 74-82.

7. Rai G. D. An introduction to power plant technology. $3^{\text {rd }}$ ed. Delhi Khanna publishers, 1997.

8. Пипченко А. Н., Пономаренко В. В., Шевченко В. А., Табулинский И. Н. Эксплуатация и ремонт одно- и двухтопливных двигателей производства Wartsila в энергетических установках морских транспортных средств: монография. Одесса: ТЭС, 2018. $361 \mathrm{c}$.

9. El-Gohary M. Morsy. Overview of past, present and future marine power plants. Journal of Marine Science and Application. 2013. No. 12 (2). P. 219-227.

10.Циннер К. Наддув двигателей внутреннего сгорания / пер. с нем. В. И. Федышина; под ред. Н. Н. Иванченко. Ленинград: Машиностроение, Ленингр. отд., 1978.

11.Шевченко В. А. Верификация функциональных алгоритмов системы управления многоагрегатной судовой электрической станцией. Автоматизащия судовых технических средств: науч.-техн. сб. 2015. Вып. 21. С. 70-76.

12.Шевченко В. А. Способ верификации функциональных алгоритмов системы управления многоагрегатной судовой электрической станцией. Науковий вісник
Херсонської державної морської академії: науч.-техн. сб. 2015, Вып. 1. С. 296-301.

\section{References}

1. Mukund R. Patel (2012) Shipboard electrical power systems. CRC Press. $370 \mathrm{p}$.

2. Kithsiri, U. G., Senanayake1, N. S., Abeyweera, Ruchira, Jayasuriya, Jeevan (2016) A model for estimation of de-rating in diesel engines used for power generation. International Journal of Energy Engineering, 6 (2), pp. 3642. DOI: 10.5923/j.ijee.20160602.03.

3. Saber, Hindren A., Al-Barwari, Ramzi R. Ibraheem, Talabany, Ziyad J. (2013) Effect of ambient air temperature on specific fuel consumption of naturally aspirated diesel engine. Journal of Science and Engineering, Vol. 1 (1), pp. 1-7.

4. Mollenhauer, Klaus, Tschoeke, Helmut (eds) (2010) Diesel engine engineering hand book. Springer, ISBN-10: 3540890823.

5. McCrone, A. K. L. Diesel plant operator's hand book. Pitman, ISBN-10: 0273409603.

6. Zakharchenko, V. N. Shevchenko, V. A. (2015) Solving of tasks for control by ship's electrical power plant in power changing conditions. Sudovyie energeticheskie ustanovki: sci.-tech. coll. Iss. 36, pp. 74-82 [in Russian].

7. Rai, G. D. (1997) An introduction to power plant technology, $3^{\text {rd }}$ ed. Delhi Khanna publishers.

8. Pipchenko, A. N., Ponomarenko, V. V., Shevchenko, V. A., Tabulinsky, I. N. (2018) Oneand dual-fuel Wartsila engines maintenance and repair in marine power plants: monograph. Odessa: TES. 361 p. [in Russian].

9. El-Gohary M. Morsy (2013) Overview of past, present and future marine power plants. Journal of Marine Science and Application. 12 (2), pp. 219-227.

10.Zinner, K. (1978) Internal combustion engines turbocharging. In: N. N. Ivanchenko (ed.). Leningrad: Mashynostroyeniye [in Russian].

11. Shevchenko, V.A. (2015) Verification of functional algorithms of control system of multiunit ship's electrical power plant. Avtomatizatsiya sudovyih tehnicheskih sredstv: sci.-tech. coll. Iss. 21, pp. 70-76 [in Russian].

12.Shevchenko, V. A. (2015) The method of verification of functional algorithms of control system of multiunit ship's electrical power plant. Naukovyi visnyk Khersonskoi derzhavnoi morskoi akademii: sci.-tech. coll. Iss. 1, pp. 296-301 [in Russian]. 
V. A. Shevchenko, Ph.D., associate professor

the head of the ships' technical maintenance department

e-mail:vash4891@gmail.com

National University "Odesa maritime academy"

Didrikhson str., 8, Odesa, 65000, Ukraine

\title{
OPTIMIZATION OF POWER PLANT CONTROL ON MINIMUM FUEL CONSUMPTION TAKING INTO ACCOUNT WEATHER CONDITIONS
}

Modern automated marine electrical power systems have low generated power load factor and, as the result, low efficiency. It can be explained not only by power plant configuration but also imperfect setup of power plant control modes. So far such tasks are being solved using approximate and in some cases rough methods tending to increase power redundancy in main ship operation modes.

During the calculation of power and load limits of power plant usually mean or critical values of uncontrollable variables (power demand, pressure, temperature and ambient humidity) are used without taking into account the changes in such parameters. The most specific changes in parameters when ship sails in tropics are the following: reduction of engine power, increase of specific fuel consumption and exhaust gas temperature caused by decrease of air weight charge and increase of its temperature.

The goal of this research is to work out the algorithms of calculation and continuous control of permissible power plant engine loads as well as the algorithms of automatic control of engine operating parameters in accordance with changes of ambient parameters.

Power plant internal combustion engines air to fuel ratio calculation method is proposed in the paper taking into account wet and dry air consumption which can be defined using given nomograms. Fuel-air ratio optimization algorithms are developed. Power plant control optimization algorithms on minimum fuel consumption taking into account weather conditions are synthesized.

The fulfilled research allows to develop marine power plant control systems, optimal during changes in load, generator engines technical condition and weather condition.

Keywords: electrical power plant control, air-fuel ratio, algorithms synthesis, fuel consumption, optimization.

\author{
В. А. Шевченко, к.т.н., доиент, \\ зав. кафедри технічної експлуатації суден \\ e-mail: vash4891@gmail.com \\ Національний університет «Одеська морська академія» \\ вул. Дідріхсона, 8, м. Одеса, 65000, Україна

\section{ОПТИМІЗАЦІЯ УПРАВЛІННЯ ЕЛЕКТРОСТАНЦЕЮ З УМОВИ МІНІМУМУ ВИТРАТ ПАЛИВА І 3 УРАХУВАННЯМ МЕТЕОУМОВ} \\ Сучасні суднові електроенергетичні системи мають низький коефіиієнт завантаження \\ $i$, як наслідок, низький коефіцієнт корисної дії. Це може бути наслідком не тільки невдалої \\ конфігураиії електроенергетичної установки, але й недовершеної організації ї̈ режимів робо- \\ ти. Досі вирішення завдань, що розглядаються, виконують за допомогою приблизних, а часті- \\ ше достатньо грубих способів, прагнучі підвищити резервування електричної потужності у \\ головних режимах роботи судна. При розрахунку потужності та припустимих значень заван- \\ таження електростанції за режимами зазвичай відштовхуються від усереднених або гранич- \\ них значень змінних, що не керуються (запит завантаження, температура, тиск, вологість \\ навколишнього середовища та ін.). Найбільш характерними змінами параметрів при плаванні \\ судна у тропічних широтах є зменшення потужності двигунів, підвищення витрати пального
}


та температури вихлопних газів, викликані зменшенням заряду повітря та зростанням його температури. Метою цьвого дослідження є вирішення завдання управління електростанцією з умов мінімуму витрати пального, розробка алгоритмів розрахунку та безперервного контролю припустимих навантажень дизелів, а також алгоритмів автоматичного регулювання параметрів роботи двигуна з урахуванням змін навколишнього середовища. У роботі проаналізовано режими роботи електростанцій сучасних суден та їх середнє завантаження. Запропоновані вирази для визначення сумарного коефіцієнта надлишку повітря двигунів у судновій електростанції з урахуванням витрати вологого та сухого повітря, що визначається за номограмами, наданими у роботі. Отримано вирази, які дозволяють визначити оптимальний коефіцієнт надлишку повітря двигуна внутрішнього згоряння, що відповідає максимальному ККД. Розроблено алгоритми управління електростанцією із урахуванням мінімуму витрати палива шляхом варіювання коефічієнта надлишку повітря та подачі палива. Виконані дослідження дозволяють встановити правила функиіонування системи оптимального управління електростаниією при зміні завантаження, технічного стану генераторних агрегатів та умов навколишнього середовища.

Ключові слова: управління електростаниією, коефіцієнт надлишку повітря, синтез алгоритмів, витрата палива, оптимізація. 